Disclosures A. Spiotta: 1; C; Stryker, Penumbra, Medtronic. 2; C; Stryker, Penumbra, Terumo. C. Schirmer: None. B. Bohnstedt: None. R. Bellon: None. H. Hawk: None.

\section{P-049 ELECTIVE INTERVENTION FOR UNRUPTURED CRANIAL ARTERIOVENOUS MALFORMATIONS IN RELATION TO ARUBA TRIAL: A NATIONAL INPATIENT SAMPLE STUDY}

${ }^{1} \mathrm{~W}$ Wahood*, ${ }^{2} \mathrm{~A}$ Alexander, ${ }^{3} \mathrm{R}$ Doherty, ${ }^{4} \mathrm{~A}$ Bhandarkar, ${ }^{2} \mathrm{G}$ Lanzino, ${ }^{2} \mathrm{M}$ Bydon, ${ }^{2}$ W Brinjikji. 'Nova Southeastern University College of Allopathic Medicine, Davie, FL; ${ }^{2}$ Department of Neurosurgery, Mayo Clinic, Rochester, MN; ${ }^{3}$ School of Medicine, Trinity College Dublin, University of Dublin, Dublin, Ireland; ${ }^{4}$ Alix School of Medicine, Mayo Clinic, Rochester, MN

\subsection{6/neurintsurg-2021-SNIS.85}

Background In 2014, A Randomized Trial of Unruptured Brain Arteriovenous Malformations (ARUBA) concluded that medical management alone for cranial arteriovenous malformations (AVMs) had better clinical outcomes than interventional treatment. The impact of the ARUBA study on changes in the rates of intervention and outcomes is unknown. Thus, we investigated whether the conclusions from ARUBA may have influenced treatment modalities and outcomes of unruptured AVMs.

Methods The National Inpatient Sample (NIS) was queried between 2006 to 2018, for adult patients with an AVM who were admitted on an elective basis. Interventions included open, endovascular, and stereotactic surgeries. Logistic regression was conducted to assess treatment rate for each modality before and after the time-point, odds of non-routine discharge and hemorrhage between the two time-points. Linear regression was used to assess mean LOS between the two timepoints.

Results A total of 40,285 elective admissions for AVMs were identified between 2006 and 2018. The rate of intervention was higher pre-ARUBA $(n=15,848 ; 63.8 \%)$ compared to postARUBA $(n=6,985 ; 45.2 \% ; p<0.001)$. The rate of open, endovascular, and stereotactic surgeries decreased after the ARUBA Trial time-point (ORs: 0.37, 0.69, and 0.18, respectively; $\mathrm{p}<0.001$ ). For admissions involving interventions, the odds of non-routine discharge were higher post-ARUBA (OR: 1.24; $\mathrm{p}=0.043)$; the odds of hemorrhage were lower post-ARUBA (OR: $0.69 ; \mathrm{p}=0.025$ ). There was no statistical difference in length of stay between the two time-points $(p=0.22)$.

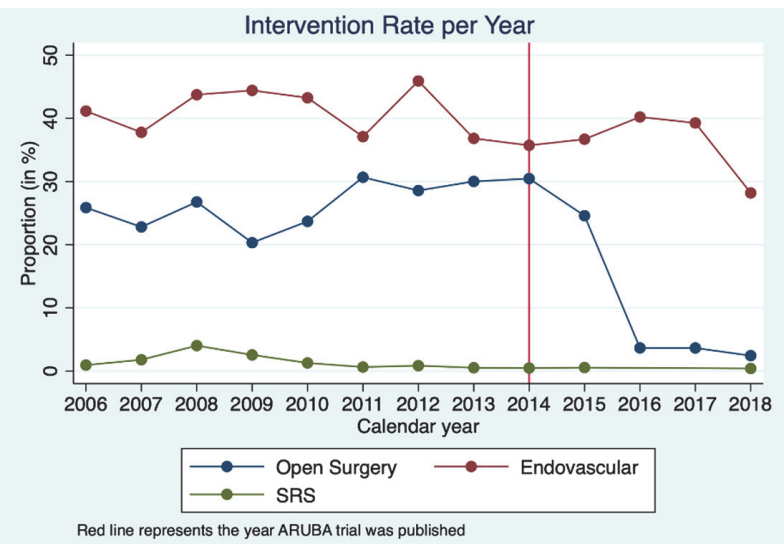

Abstract P-049 Figure 1
Conclusion The rate of intervention decreased, the rate of non-routine discharge increased, and rate of hemorrhage decreased post-ARUBA, suggesting that it may have influenced treatment practices for unruptured AVMs.

Disclosures W. Wahood: None. A. Alexander: None. R. Doherty: None. A. Bhandarkar: None. G. Lanzino: None. M. Bydon: None. W. Brinjikji: None.

\section{P-050 RECENT OUTCOMES FOLLOWING ENDOVASCULAR VS OPEN SURGICAL TREATMENT OF RUPTURED AND UNRUPTURED CEREBRAL ANEURYSM: INSIGHTS FROM THE NATIONAL INPATIENT SAMPLE 2017}

${ }^{1} \mathrm{H}$ Saber*, ${ }^{2} \mathrm{M}$ Jafari, ${ }^{2} \mathrm{~A}$ Desai, ${ }^{1} \mathrm{~N}$ Kaneko, ${ }^{3} \mathrm{G}$ Colby, ${ }^{1} \mathrm{~V}$ Szeder, ${ }^{1} \mathrm{R}$ Jahan, ${ }^{1} \mathrm{G}$ Duckwiler, ${ }^{1} S$ Tateshima. IInterventional Neuroradiology, UCLA, Los Angeles, CA; ${ }^{2}$ Neurology, Baylor College of Medicie, Houston, $T X_{;}{ }^{3}$ Neurosurgery, UCLA, Los Angeles, CA

\subsection{6/neurintsurg-2021-SNIS.86}

Introduction/Background Advancements in endovascular therapies have significantly impacted cerebral aneurysm management in recent years. Recent release of the National Inpatient Sample (NIS) data for 2017 provides the opportunity to explore novel population-based insights into outcomes following surgical clipping vs endovascular embolization of ruptured and unruptured cerebral aneurysms.

Materials and Methods This is an analysis of US National Inpatient Sample of hospitalizations with non-traumatic subarachnoid hemorrhage (SAH) or unruptured cerebral aneurysms from January 1, 2017 to December 31, 2017. Baseline patient demographics, open vs endovascular treatment modalities and the associated hospitalization outcomes were analyzed. Primary outcomes included in-hospital mortality and favorable discharge disposition defined as discharge to home.

Results Among 56,165 hospitalizations with unruptured aneurysms, 13,090 (23.3\%) underwent endovascular embolization (median age 59, IQR 51-68; Female 74.9\%) and 4105 (7.3\%) had surgical clipping (median age 58, IQR 51-65; Female $74.1 \%$ ). In-hospital mortality occurred in $1.45 \%$ in endovascular vs $1.83 \%$ in clipping group $(p=0.17)$, whereas favorable discharge outcome was achieved in $87.5 \%$ in endovascular vs $74.8 \%$ in clipping group $(\mathrm{p}<0.001)$. Median hospital length-of-stay was 1 day (IQR 1-4) in endovascular vs 4 days (IQR 3-8) in clipping group $(\mathrm{P}<0.001)$. Significantly more favorable outcomes were achieved with coiling vs clipping in those aged 65 or above with unruptured aneurysms. Among 41,420 hospitalizations with non-traumatic $\mathrm{SAH}$, $6,400(15.5 \%)$ underwent endovascular embolization and 2,185 (5.3\%) had surgical clipping. In-hospital mortality occurred in $12.6 \%$ in endovascular vs $13.9 \%$ in clipping group $(p=0.41)$, and favorable discharge outcome was achieved in $44.8 \%$ in endovascular vs $40.1 \%$ in clipping group $(p=0.1)$ with $\mathrm{SAH}$. Median length-of-stay was 15 days (IQR 10-22) in endovascular vs 16 days (IQR 11-22) in clipping group $(\mathrm{P}=0.7)$ with $\mathrm{SAH}$. Clipping was more frequently utilized in younger population (50 years or younger) with $\mathrm{SAH}$; however, outcomes were more favorable with coiling vs clipping in this subgroup (mortality $7.7 \%$ vs $11.8 \% ; \mathrm{p}=0.01$, respectively).

Conclusion In 2017 in US, about 75\% of patients were discharged home after clipping and 90\% were discharged home after endovascular treatment of an unruptured cerebral aneurysm. About 1 in 7 patients died following clipping and 1 in 8 following endovascular treatment for aneurysmal SAH. 
Endovascular therapy remains associated with better in-hospital outcomes, including the younger age population with ruptured or unruptured aneurysms.

Disclosures H. Saber: None. M. Jafari: None. A. Desai: None. N. Kaneko: None. G. Colby: 2; C; Medtronic, Stryker, MicroVention. V. Szeder: None. R. Jahan: 2; C; Medtronic. G. Duckwiler: 2; C; Medtronic. S. Tateshima: 2; C; Medtronic, Stryker, Cerenovus.

\section{P-051 TREATMENT OF ACUTELY RUPTURED INTRACRANIAL ANEURYSMS WITH WOVEN ENDOBRIDGE DEVICE: A SYSTEMATIC REVIEW}

${ }^{1} \mathrm{~A}$ Monteiro*, ${ }^{2} \mathrm{~A}$ Lazar, ${ }^{1} \mathrm{M}$ Waqas, ${ }^{1} \mathrm{H}$ Rai, ${ }^{1} \mathrm{~A}$ Baig, ${ }^{1} \mathrm{R}$ Dossani, ${ }^{1} \mathrm{~F}$ Almayman, IJ Cappuzzo, 'I Davies, ${ }^{1} \mathrm{~K}$ Snyder, ${ }^{1} \mathrm{E}$ Levy, ${ }^{1} \mathrm{~A}$ Siddiqui. ${ }^{1}$ Neurosurgery, University at Buffalo Neurosurgery, Buffalo, NY; ${ }^{2}$ Neurosurgery, Gates Vascular Institute at Kaleida Health, Buffalo, NY

\subsection{6/neurintsurg-2021-SNIS.87}

Introduction The Woven Endobridge (WEB) device is a barrelshaped nitinol mesh deployed within the aneurysmal sac. The absence of metallic mesh in the vessel lumen obviates the need for potent antiplatelet therapy, which makes this devices interesting for acutely ruptured aneurysms not amenable to clipping or coiling.

Methods We performed a comprehensive systematic search of Pubmed, MEDLINE and EMBASE databases following the Preferred Reporting Items for Systematic Reviews and MetaAnalyses (PRISMA) guidelines. Keywords combined with Boolean operators to increase search sensitivity and specificity ('woven endobridge device' AND 'ruptured') were used.

Results Five studies were included, totalizing 276 ruptured aneurysms. Overall, $222(80.4 \%)$ were wide-neck and 236 $(85.5 \%)$ were located in the anterior circulation. Grade of SAH based on Hunt and Hess scale was reported in four studies with $64(25.7 \%)$ of patients presenting with a poor grade (Hunt and Hess 4-5). Intraoperative and post-operative complications occurred in 7.2\% (95\% CI, 3.8-13.4) and $4.3 \%$ (95\% CI, 1.9-9.2) respectively. The rate of rebleeding after treatment was $0 \%$. The rates of adequate occlusion and retreatment at last follow-up were $86.5 \%$ (95\% CI, 75.6-93) and $5.9 \%$ (95\% CI, 3.2-10.6), respectively. The rate of favorable outcome was $71 \%$ (95\% CI, 64-77) and mortality was $19.4 \%$ (95\% CI, 14.3-25.7). The United-States multicenter study reported use of intraoperative single-antiplatelet therapy (SAPT) in $13.1 \%$ and dual-antiplatelet therapy (DAPT) in $2.2 \%$, while $18.7 \%$ were discharged under DAPT. The remaining 4 European studies reported no use of antiplatelets during the procedure unless stenting was needed. Two studies reported no use of antiplatelet regimen during follow-up, while 2 studies reported use of SAPT for 4-6 weeks.

Conclusions Treatment of acutely ruptured aneurysms with WEB device results in high rates of adequate occlusion, with low perioperative complications, no rebleeding and low recurrence. This device is promising for wide-necked ruptured aneurysms that are not amenable to clipping or coiling, considering its lower need for antiplatelet regimen either during procedure or follow-up.

Disclosures A. Monteiro: None. A. Lazar: None. M. Waqas: None. H. Rai: None. A. Baig: None. R. Dossani: None. F. Almayman: None. J. Cappuzzo: None. J. Davies: 1; C; National Center for Advancing Translational Sciences of the National Institutes of Health under award number
KL2TR001413 to the University at Buffalo. K. Snyder: 2; C; Canon Medical Systems Corporation, Penumbra Inc., Medtronic, and Jacobs Institute. E. Levy: 2; C; Claret Medical, GLG Consulting, Guidepoint Global, Imperative Care, Medtronic, Rebound, StimMed;. 4; C; NeXtGen Biologics, RAPID Medical, Claret Medical, Cognition Medical, Imperative Care (formerly the Stroke Project), Rebound Therapeutics, StimMed, Three Rivers Medical;. A. Siddiqui: 2; C; Amnis Therapeutics, Boston Scientific, Canon Medical Systems USA Inc., Cerebrotech Medical Systems Inc., Cerenovus, Corindus Inc., Endostream Medical Ltd., Imperative Care, Inc. Integra LifeSciences C. 4; C; Adona Medical, Inc, Amnis Therapeutics, (Purchased by Boston Scientific October 2017), Blink TBI Inc., Buffalo Technology Partners Inc., Cerebrotech Medical Systems, Inc., Cognition Medical, Endostrea.

\section{P-052 A NOVEL EX-VIVO MODEL TO SIMULATE DELAYED ANEURYSM RUPTURE AFTER FLOW-DIVERTER TREATMENT}

${ }^{1} \mathrm{H}$ Sodawalla*, ${ }^{1} \mathrm{~W}$ Merritt, ${ }^{2} \mathrm{G}$ Uyeda, ${ }^{3} \mathrm{~T}$ Becker. ${ }^{1}$ Bioengineering Program (Department of Mechanical Engineering), Northern Arizona University, Flagstaff, $A Z ;{ }^{2}$ Department of Applied Physics and Materials Science, Northern Arizona University, Flagstaff, $A Z ;{ }^{3}$ Bioengineering Program (Department of Mechanical Engineering), Northern Arizona University, Flagstaff, AZ

\subsection{6/neurintsurg-2021-SNIS.88}

Introduction/Purpose It is estimated that 3-6 million Americans harbor a brain aneurysm and are at risk for hemorrhagic stroke (rupture). Flow-diverter placement in the blood vessel (fine mesh stent placement) across giant intracranial aneurysms (GIAs) is the standard of care. However, up to $20 \%$ of these aneurysms still experience delayed aneurysm rupture (DAR). Intra-aneurysmal thrombus formed after a flow-diversion treatment releases matrix metalloproteinase (MMPs) that can degrade the extracellular matrix of an aneurysmal wall and cause delayed rupture. Current in vivo aneurysm models (i.e., swine/canine vein pouch-to-artery anastomosis models and rabbit-elastase models) result in relatively stable aneurysms and do not model giant aneurysms, nor do they simulate the tissue degradation preceding DAR. This study evaluated the mechanobiological properties of mouse arterial vessel wall digestion by MMP- $1 / 2 / 9$ proteins with the aim of creating a novel exvivo DAR model.

Materials and Methods Abdominal aorta tissue was harvested from homozygous inbred female mice (The Jackson Laboratory, Bar Harbor, Maine) within 3 hours of sacrifice and flushed with phosphate buffered saline. The tissue samples were then divided into 5 groups ( $\mathrm{n}=4$ each for group): porcine pancreatic elastase (PPE) treated group, MMP-1 group, MMP-2 group, MMP-9 group, and a cocktail group (3 MMPs in equal quantities). Each group includes three trials per group - control trial, low dose trial $(0.2 \mathrm{ug} / \mathrm{ml})$ and high dose trial (2 $\mathrm{ug} / \mathrm{ml})$. The three trial groups were tested for macroscopic mechanical properties, using non-destructive testing protocols for shear modulus, compression modulus and tensile modulus, with NAU's HR-2 rheometer (TA Instruments, New Castle, DE). Testing was conducted before (control) and after incubation for 2 hours at $37^{\circ} \mathrm{C}$. For microscopic mechanical analysis, prior to tissue fixation, the tissue samples were visualized with confocal microscopy to determine changes in its arterial structure and atomic force microscopy (AFM) to quantify enzyme tissue digestion. 